\title{
PECULIARITIES OF INFLUENCE OF WELDING AEROSOLS ON THE RESPIRATORY SYSTEM
}

\section{Kashuba M. 0.}

\section{Horbachevsky Ternopil National Medical University}

Introduction. Respiratory diseases of electric welders are a common occupational disease. The cause of these diseases is associated with the effects of welding aerosols on the respiratory system. One of the important properties of aerosols, on which depends the peculiarity of their effect on the respiratory system, is their dispersion. Therefore, the study of the dispersed composition of welding aerosols entering the respiratory system is an important point for understanding the peculiarities of the formation of pneumoconiosis.

Purpose of research. To investigate the aerodynamic features of welding aerosols and their permeability to the respiratory system.

Materials and methods of research. The particle size of welded aerosols was studied by means of optical and electron microscopy. Sampling from the air of the working area was carried out with the patented «Method of determining dust air pollution in the premises». Sampling of inhaled and exhaled aerosol was performed with the device for determining the absorbed dose of aerosol.

Results. Dust particles of different sizes both in quantitative terms and by weight are distributed on separate sites of respiratory organs as follows. Fractions up to $0.5 \mu \mathrm{m}$ in size are mostly represented in the alveoli, fractions from 0.5 to $8.6 \mu \mathrm{m}$ in size are mostly delayed in the larynx, trachea, bronchi, and particles larger than $8.6 \mu \mathrm{m}$ are found in the mouth cavity and nasopharynx. Aerosol fractions with a sedimentation radius (Rs) of 10.4-12.3 $\mu \mathrm{m}$ make up the largest part of the total mass of dust deposited in the oral cavity and pharynx, fractions with Rs of 4.6-6.5 $\mu \mathrm{m}-$ in the larynx, trachea, bronchi, and fractions with Rs $0.3-0.6 \mu \mathrm{m}-$ in the alveoli.

Conclusions. Under conditions of use in the production of air recirculation system, the total weight concentrations of the welding aerosol do not reflect the actual effect of dust on the respiratory system. It would be more correct under these conditions to evaluate the effect of the aerosol on the respiratory system by the size of its respirable fraction.

Key words: aerosols, weight concentrations, sedimentation, pneumoconiosis

\section{Introduction}

The intensity of respiratory exposure to welding aerosols is in most cases assessed by their concentration in workplace air. In some countries the respirable fraction is also used to assess their effects. However, the latter is also not an adequate indicator of the effects of aerosols on all parts of the respiratory system. To a greater extent, it reflects with relative accuracy only the effects on the lungs.

The intensity of human exposure to aerosol depends not only on its concentration in the air of working area, but also on the properties of dif- ferent dust fractions [1-4] and their ability to penetrate into the respiratory system. This, in its turn, depends not only on physical parameters of particles, but also on anatomical and physiological features of the respiratory system, peculiarities of the work performed (peculiarities of welding [5] and a number of other factors). However, the role of all these factors has not been sufficiently studied.

The aim was to study the physical and, in particular, aerodynamic features of welding aerosols and the influence of these features on their penetration into the respiratory system. 
In this regard, the capability of particles of various sizes to be retained in certain parts of the respiratory tract was studied using the methods developed by us.

\section{Materials and methods of research}

The study of physical parameters of aerosols was carried out using optical and electron microscopy methods, cameras with particle counting grids, devices for determining physical parameters of micro-particles in the environment [6], method for determining physical parameters of gas-dust environment [7], Method for determination of dust pollution in indoor air [8], as well as sampling and research of nanoparticles in workplace air [9, 10]. Investigation of microparticle penetration into respiratory organs was carried out with devices for determination of sorption capacity of aerosols [11] and «devices for determination of absorbed dose of aerosol» [12].

With regard to the set task, we studied the dispersed composition and mass of different fractions of welding aerosols deposited in the mouth (nose), respiratory tract and lungs, based on the quantitative composition and mass of particles of different fractions, exhaled from these areas.

Studies have shown that the quantitative composition of inhaled aerosols differs from that of aerosols in workplace air. Fig. 1 of the logarithmic $X$ and $Y$ scales shows the dispersed composition of dust in the air of the work area (total airborne dust) and the dust inhaled.

As can be seen in the graph, with increasing particle size the difference in their quantity in the inhalable air, and in the air of industrial premises increases. The linear nature of the graphs indicates that there is a correlation between the particle sizes and their quantitative composition both in the inhaled air and in the working air.

The nature of this dependence is explained as follows. Settling of particles is determined by gravity $(\mathrm{mg})$, which is proportional to the cube of the particle radius, i.e. $\mathrm{F}_{\text {gravity }} \sim \mathrm{R}^{3}$. On the other hand, the settling of the particles is counteracted by the force of air resistance, which is proportional to the velocity of the particle $(\mathrm{v})$, and its linear dimensions $(\mathrm{R}), \mathrm{F}_{\text {resistance }} \sim \mathrm{V} \mathrm{R}$.

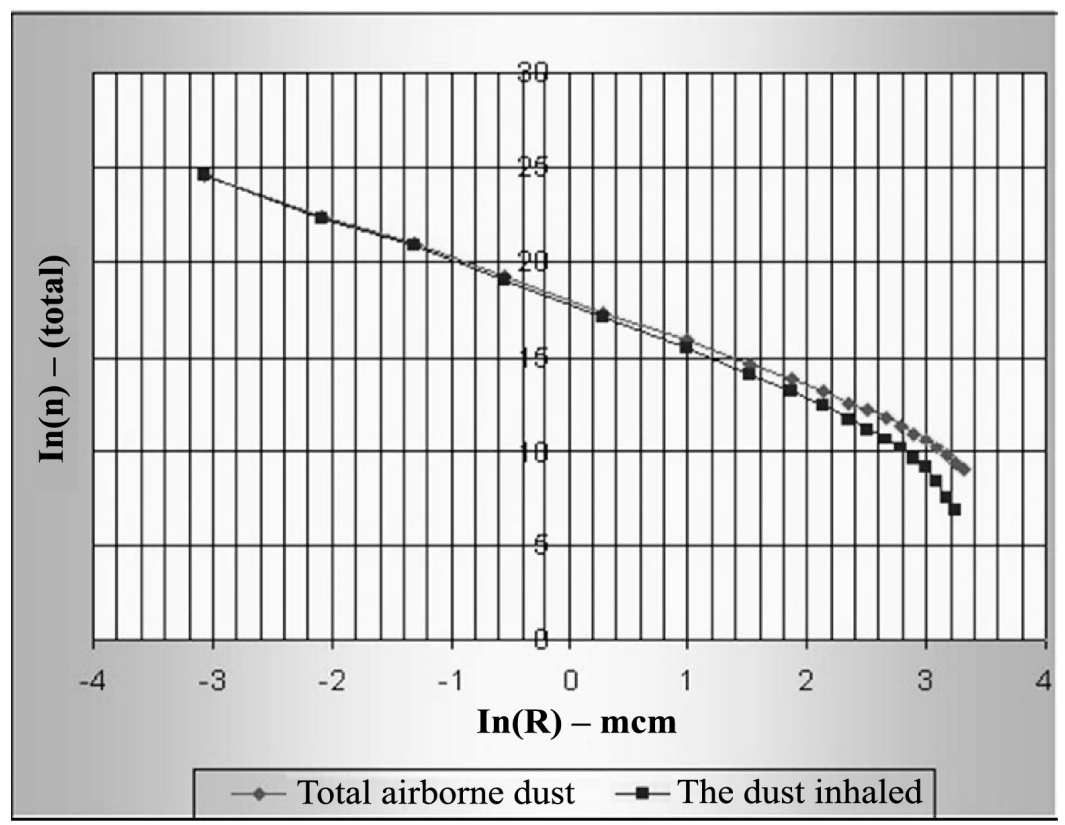

Fig. 1. Quantitative composition of dust particles of different aerosol fractions in workplace air and inhalable aerosol ( $X$ and $Y$ scales are logarithmic) 
Comparison of Fgravity and $\mathrm{F}_{\text {resistance }}\left(\mathrm{R}^{3} \sim \mathrm{vR}\right)$ gives average settling velocity of the particles $\left(\mathrm{v} \sim \mathrm{R}^{2}\right)$. The concentration of particles $\left(\mathrm{n}_{1}\right)$ of a given size in the air can be determined by comparing the number of particles deposited from the air per unit time $\left(\mathrm{N}_{2} \sim \mathrm{nlv}\right)$ with the concentration of particles generated by a pollution source per unit time $\left(\mathrm{N}_{3}\right)$. Generation of particles of a given size is a constant. Based on the above, $n_{1} V^{\sim} G(R)$, where $G(R)$ is the number of particles of size $\mathrm{R}$ generated in the air. Hence $\mathrm{n}_{1} \sim$ $\mathrm{G}(\mathrm{R}) / \mathrm{v}$, or $\mathrm{n} 1 \sim \mathrm{G}(\mathrm{R}) \mathrm{R}^{-2}$. (1/v). That is, the change in the concentration of particles in the air $\left(n_{1}\right)$ is proportional to $R^{-2}$. Particle settling time $(t)$ is inversely proportional to its velocity $t \sim 1 /$ $\mathrm{R}^{2}$ or $\sim \mathrm{R}^{-2}$, so $\left(\mathrm{n}_{1}\right)$ is also proportional to $\mathrm{t}$.

This kind of dependence is in fact reflected in Fig. 1 in graphs of distribution of particles of different sizes in the total mass of aerosol as a function of their settling velocity, which is conditioned by their sizes and masses.

However, as pointed out by some researchers, dust dispersion composition depends on the distance from the dust source, at which measurements were made, and on time which has passed since the dust emission [13].

As can be seen from Fig. 1, the number of particles with a sedimentation radius (Rs) of up to $1 \mu \mathrm{m}$ in inhalable air is almost the same as in workplace air. So, particles of this size are almost unimpeded in the respiratory system.

As can be seen from the graph, the line that represents the amount of dust particles inhaled increasingly deviates from the similar graph for total swirling dust from a size of $1 \mu \mathrm{m}$ or more. This indicates that with increasing particle size the ability to penetrate the respiratory tract decreases.

Sampling and conimetric studies of the dispersed composition of dust particles, which are exhaled from different parts of the respiratory system, were carried out using an instrument for determining the absorbed dose of aerosol.
Based on the results of the study, the dispersion composition of dust deposited in the respiratory tract and lungs was determined by calculation. Calculations were made using appropriate formulas developed by us. The results are shown in Fig. 2.

The mass of the dust fraction was calculated taking into account the shape factor using the formula:

$$
\mathrm{m}=4 / 3 \pi \mathrm{R}_{\mathrm{p}}^{3} \rho \mathrm{Kfn},
$$

where $\rho$ - specific weight of dust; $R_{r}-$ projection radius of the particle; $\mathrm{Kf}-$ shape factor; $\mathrm{n}$ - number of particles.

The results are shown in Fig. 3, where the mass of individual dust fractions deposited at different respiratory areas, as well as dust inhaled and total hovering dust are presented.

Particles of different sizes, both quantitatively and by mass, are distributed over individual's respiratory organs as follows (see Figs. 2, 3). Particles up to $0.5 \mu \mathrm{m}$ are most abundant in alveoli, particles from 0.5 to $8.6 \mu \mathrm{m}$ are more abundant in larynx, trachea, bronchi and particles over $8.6 \mu \mathrm{m}$ in oral cavity and nasopharynx. This shows that certain parts of the respiratory organs have a selective capacity to trap particles of certain sizes.

As shown in Fig., the aerosol fractions with sedimentation radius (Rs) of $10.4-12.3 \mu \mathrm{m}$ constitute the largest part of the total dust mass deposited in the oral cavity and pharynx, the fractions with Rs 4.6-6.5 $\mu \mathrm{m}$ - dust deposited in respiratory tract (larynx, trachea, bronchi), and fractions with Rs $0.3-0.6 \mu \mathrm{m}-$ dust deposited in alveoli. In quantitative terms, fine particles are found in all parts of the respiratory tract. Total weight of dust, settled in respiratory organs, is $18.4 \%$ of total particles, and $54.1 \%$ of all that is inhaled. Thus, in weight concentrations the fractions with Rs $10.4-12.3 \mu \mathrm{m}$ are the most dangerous for upper airways; for trachea-bron- 


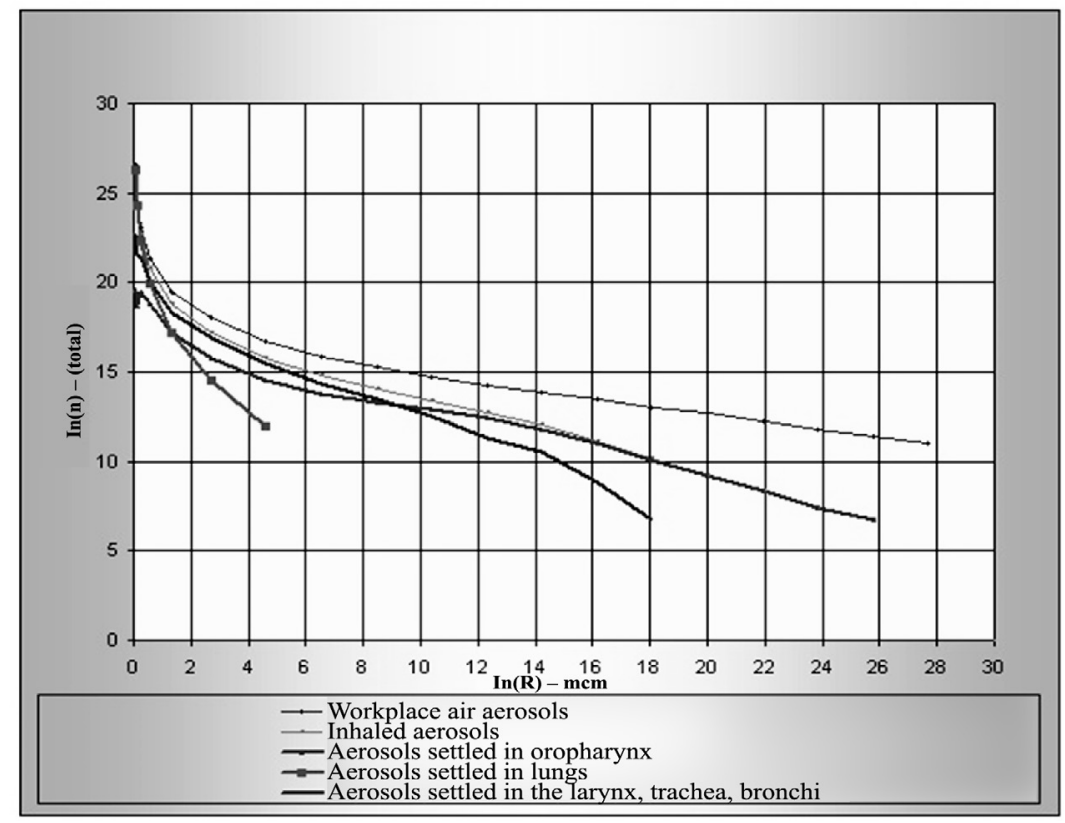

Fig. 2. Quantitative composition of fractions of different sizes, which settled in certain areas of the respiratory system

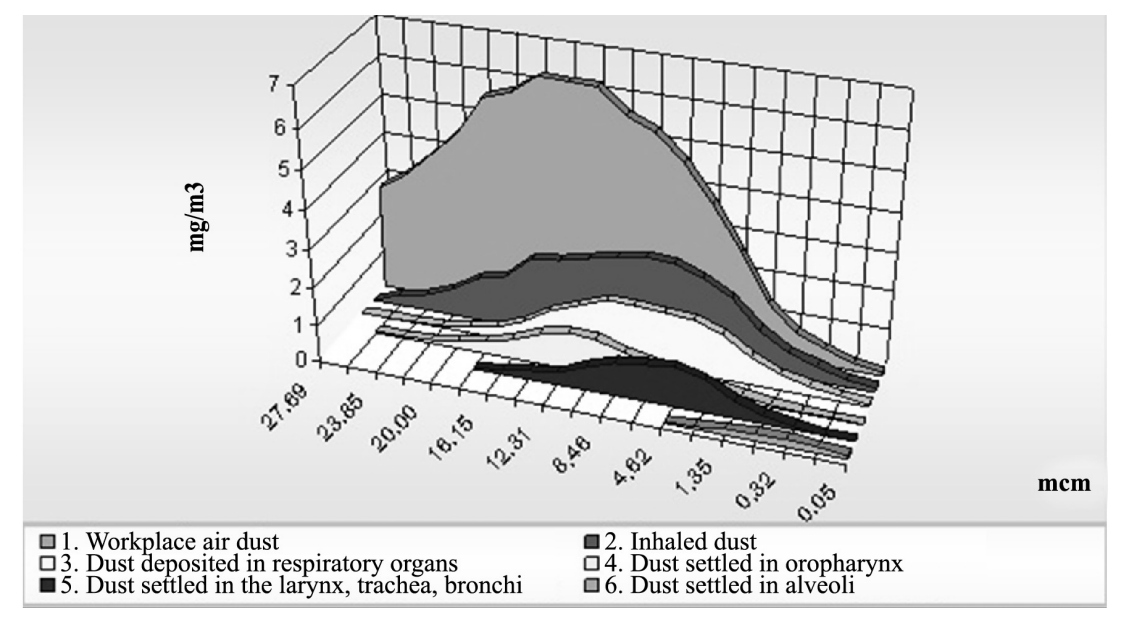

Fig. 3. Mass of different aerosol fractions in workplace air, aerosol inhaled and aerosol deposited at individual's respiratory organs

chial part - with Rs 4.6-6.5 $\mu \mathrm{m}$; for alveoli with Rs $0.3-0.6 \mu \mathrm{m}$.

Calculations showed that mass of dust, settled in respiratory organs, is not divided in its parts in regular intervals. Nasopharynx absorbed $47.9 \%$ of dust mass, larynx, trachea and bronchi $-45.4 \%$, alveoli $-6.7 \%$. Even more uneven distribution of dust mass is observed at its distribution per unit volume of the studied section of respiratory organs. In larynx, trachea and bronchi 2.1 times less dust mass is settled per unit volume than in oropharynx, and in alveoli - 57 times less than in oropharynx and 27 times less than in larynx, 
trachea and bronchi. This fact testifies not only to higher protection of lungs against dust due to higher sections which play the role of peculiar filters, but also to the fact that dust load on lungs increases sharply in case the filtration ability of airways decreases.

It is known that particles with radius less than 1 micron penetrate into alveoli in great amount. It is obvious, that with the growth of dustiness of working zone air the usage of different collective and individual respiratory protection means increases. However, it is known that their ability to trap dust particles decreases with the decrease of particle size. Therefore, after the aerosol has passed through the filter, the proportion of fine particles in the total of aerosol particles will increase. Thus, although the total weight concentration of aerosol due to apprehension on filters of coarse particles will be significantly reduced, the quantitative composition of the particles will not change significantly.

Presence of powerful air pollution sources in workshops and consequent use of recirculation

\section{References}

1. Rakov Yu. V., Smolina A. S., Kuznetsov D. A. et al. (2014), "About the classification and some physical and chemical properties of industrial and welding dust and aerosols", Master's Journal: Perm National Research Polytechnic University, 1, 53-61.

2. Kirichenko K. Yu., Drozd V. A., Chaika V. V. et al. (2015), "Welding aerosol as a source of hazardous to health man-made nanoparticles: granulometric analysis», Proceedings of the Samara Scientific Center, 17, 662-665.

3. Zhang M., Jian L., Bin P. et al. (2013), "Workplace exposure to nanoparticles fromgas metal arc welding process", J. Nanopart. Res., 15, 2016. https://doi. org/10.1007/s11051-013-2016-4.

4. Borskiver I. A. (2011), «Fire arc. The Effect of Welding Spray on the Organism of the Electric Welder (Manual arc Welding). Recommendations for Measuremen", Safety and Labor Protection, 47, 2, 66-69.

5. Il'yaschenko D. P., Chinakhov D. A., Sadikov I. D. (2016), "Effect of Dynamic Characteristics of Power air cleaning system may lead to a situation when even though the aerosol concentration in the working area air after filter cleaning will be lower than MPC, but the dust load, especially on alveoli, may be higher in the workshops, where there is some excess of MPC. In these circumstances, under formal compliance with work zone air pollution standard, pneumoconiosis may form in workers.

\section{Conclusions}

In quantitative relation fine particles overexert in all parts of the respiratory system, especially in lungs, and therefore they are the main cause of pneumoconiosis development.

The cleaning system on filters with the subsequent recirculation of air in production facilities leads to reduction of weight concentrations of aerosol mainly due to the coarse fraction, and almost does not stop the particles smaller than 1 micron, which makes it ineffective in protecting workers from the development of pneumoconiosis.

Supplies on Aerosol Composition while Welding with Coated Electrodes», IOP Conference Series: Materials Science and Engineering, September, 142, 1, 2, 1-7. https://doi.org/10.1088/1757-899X/142/1/012007.

6. Kashuba M. O., Boychuk B. R. Method of determining the physical parameters of microparticles of the medium: Pat. No 47675 A Ukraine, IPC G01N 15/00. No 2001075017; declared 17.07.2001; publ. 15.07.2002, Bull. No 7 .

7. Kashuba M. O., Pashko K. O. Method for determining the physical parameters of microparticles of gas-dust medium: Pat. No 68094 A Ukraine, IPC G01N 15/14. No 2003108889; declared 01.10.2003; publ. 15.07.2004, Bull. No 7 .

8. Kashuba M. O., Fedoriv O. Ye. Method for determining dust air pollution in the premises: Pat. No 67997 Ukraine, IPC G01N 21 /21. No u201110273; declared 22.08.2011; publ. 12.03.2012, Bull. No 5 .

9. Kashuba M. O., Fedoriv O. Ye. Method for determining nanoparticles in air: Pat. No 54098 Ukraine, IPC: G01N 15/10. No u201005319; declared 30.04.2010; publ. 25.10.2010, Bull. No 20. 
ISSN 2223-6775, Ukrainian Journal of Occupational Health, 2021, 17 (3), 198-203

10. Kundiev Y. I., Trachtenberg I. M., Kashuba M. O., Fedoriv O. Ye., Dmytrukha N. M. Method for determining nanoparticles in air: Pat. No 69801 Ukraine, IPC G01N 15/00, G01N 15/02, G01N 15/06, G01N 15/10. No u201113521; declared 16.11.2011; publ. 10.05.2012, Bull. No 9 .

11. Kashuba M. O., Fedoriv O. Ye. Device for determining the sorption capacity of the respiratory system: Pat. No 65461 Ukraine, IPC A61B 5/08. No u201105260; declared 26.042011; publ. 12.12.2011, Bull. No 23.

\section{ORCID ID of the author:}

Kashuba M. O. (ORCID ID 0000-0002-3370-2805).

Information about sources of research funding: The research has been carried out within the departmental research work without external sources of funding.

Received: July 29, 2021

Accepted for publication: September 3, 2021

Contact Person: Kashuba M. O., I. Horbachevsky Ternopil National Medical University, 1, Svobody Square, Ternopil, 46001. Tel.: + 380352524788 .
12. Kashuba N. A., Ocheretinsky V. S., Mullyar I. B. Device for determining the absorbed dose of aerosol: A. S. 1533653 USSR, MKI A 61 B 5/08. No 4327203 / 28-14; app. 10/08/87; publ. 07.01.90, Bul. No 1. 7 p.

13. Kirichenko K. Yu., Rogulin R. S., Drozd V. A. et al. (2018), «Evaluation of the propagation of welding aerosol particles in the space of the welder's working zone, depending on time», Ecology of urbanized territories,. 42, 2, 42-51. 